\title{
Plant nuclear proteomics - inside the cell maestro
}

\author{
Matthias Erhardt ${ }^{1}$, Iwona Adamska' ${ }^{1}$ and Octavio Luiz Franco ${ }^{2}$ \\ 1 Department of Plant Physiology and Biochemistry, University of Konstanz, Germany \\ 2 Centre for Proteomic and Biochemical Analyses, Post-Graduate Programme in Genomic Sciences and Biotechnology, Catholic University of \\ Brasilia, Brazil
}

\author{
Keywords \\ cell culture; cellular proteomics; plant \\ nuclear bodies; plant nuclear proteome; \\ proteome comparison

\section{Correspondence} \\ O. L. Franco, SGAN Quadra 916, Módulo B, \\ Av. W5 Norte 70.790-160 - Asa Norte, \\ Brasilia-DF, Brazil \\ Fax: +556133474797 \\ Tel: +556134487220 \\ E-mail; ocfranco@gmail.com
}

doi: $10.1111 / 1.1742-4658.2010 .07748 . x$
The eukaryotic nucleus is highly dynamic and complex, containing several subcompartments, several types of DNA and RNA, and a wide range of proteins. Interactions between these components within the nucleus form part of a complex regulatory system that is only partially understood. Rapid improvements in proteomics applications have led to a better overall determination of nucleus protein content, thereby enabling researchers to focus more thoroughly on protein-protein interactions, structures, activities, and even post-translational modifications. Whereas proteomics research is quite advanced in animals, yeast and Escherichia coli, plant proteomics is only at the initial phase, especially when a single organelle is targeted. For this reason, this review focuses on the plant nucleus and its unique properties. The most recent data on the nuclear subproteome will be presented, as well as a comparison between the nuclei of plants and mammals. Finally, this review also evaluates proteins, identified by proteomics, that may contribute to our understanding of how the plant nucleus works, and proposes novel proteomics technologies that could be utilized for investigating the cell maestro.

\section{Introduction}

The nucleus is the most prominent structure within a eukaryotic cell. The organelle is clearly visible by light microscopy, and was discovered in the 17 th century by Antonie van Leeuwenhoek (1632-1723). It contains most of the DNA, organized into chromosomes, and it is the site of DNA replication and transcription. Furthermore, this organelle contains several subcompartments [1], resulting from molecular interactions. The nucleus is surrounded by a double membrane, and this constitutes a major difference between prokaryotic and eukaryotic cells. Moreover, more accurate analysis indicates a constant flux of molecules with distinct regulatory functions through the envelope, making the nucleus one of the most important regulatory organs within the cell, acting as the maestro in an enormous cell orchestra. Such models of self-organization are notoriously difficult to investigate, because it is impossible to experimentally manipulate a single component of a specific pathway without nonspecifically affecting the entire system [2].

How are we to investigate such a complex organ, which is basically defined by interactions between molecules? Until recently, microscopy and immunochemistry techniques were used to shed some light on this structure, although such techniques involve a major disadvantage, which is that they cannot identify the

\section{Abbreviations}

CB, Cajal body; DFC, dense fibrillar component; FC, fibrillar centre; LC, liquid chromatography; SILAC, stable isotope labelling by amino acids; SnRNP, small nuclear ribonucleoprotein; SR, serine/arginine rich; $2 D E$, two-dimensional gel electrophoresis. 
interactions of several molecules at the same time. For a more thorough understanding, techniques that can reveal the complex overall situation within the plant nucleus have to be applied.

In this scenario, proteomics is a rising field of research, and solves, at least partially, the problem of studying several proteins at the same time. It can be defined traditionally as the systematic analysis of the proteome, the protein complement expressed by a genome [3]. Nowadays, proteomics studies provide quantitative annotations of protein properties, including intracellular distributions, concentrations, turnover dynamics, interaction partners, and post-translational modifications [4]. Considering the sensitivity of the most recent proteomics techniques and, consequently, the enormous amount of information that is obtained, one must consider reducing the quantity of data to a feasible level. In most cases, analysis of the whole cell proteome is not helpful. Purification of compartments and subsequent analysis of subproteomes is often the only way of gaining useful information [5]. Subsequent combination of the datasets of several subproteomes can give indications about how the metabolism of the organism is regulated.

However, analysis of the proteome and the metabolome (the entirety of all metabolites within an organism) continues to pose significant challenges [6]. Considering the divergence in the plant genomic sequence (The Arabidopsis Genome Initiative [7]), cross-kingdom comparisons of the location/function of proteins are difficult to apply. The plant nucleus possesses some significant differences in appearance and composition, indicating specific molecular pathways. Hence, comparisons between mammals and higher plants, for example, have to be handled with care. It should always be remembered that a proteomic analysis can give only limited insights into the molecular orchestration within a compartment and is not a foolproof tool.

In summary, this review focuses on plant nucleus proteomes, as the proteomics of whole plants [8-13] has been previously reviewed. Furthermore, we will here discuss the uniqueness of the nucleus within the cell and the problems to be overcome when investigating this complex organelle.

\section{Nuclear structure - dynamics and differences}

The nucleus is a very complex heterogeneous structure containing several subcompartments (Fig. 1), namely the nucleolus, a chromatin-rich region composed of condensed heterochromatin, and more scattered inter- chromatin and euchromatin regions [1]. With improved microscopy techniques, about 30 different compartments [14] have recently been discovered. The uniqueness of the nucleus is shown by the fact that all of its subcompartments are membrane-less, self-organizing entities that pass through a state of disassembly/reassembly during cell division. In fact, nuclear molecules are highly dynamic and in constant exchange, and their morphology is totally determined by the functional interaction of their components [15]. The existence of this high number of intranuclear compartments is indicative of a specific location for a specific function.

The nucleus harbours two mutually interrelated structures containing nucleic acids: chromatin and the nuclear matrix [16]. The latter is a nonhistone structure that serves as a support for the genome and its activities. Calikowski et al. [17] initially characterized the Arabidopsis thaliana nuclear matrix by electron microscopy and MS. They observed a very similar structure to that described for the animal nuclear matrix.

The other nucleic acid-containing structure is chromatin, which is arranged into chromosomes. They are organized in distinct areas [18] and occupy distinct positions with respect to the periphery. It has been shown that their distribution pattern and expression profile are closely linked. Furthermore, changes in gene expression during differentiation, development and diseases can be linked to changes in genome-positioning patterns. Contributing to the whereabouts of the chromosomes, there are the matrix attachment regions on the genome, interacting with the nuclear matrix and affecting gene regulation [19]. As another example, Cajal bodies (CBs) are probably involved in small nuclear ribonucleoprotein (snRNP) and small nucleolar ribonucleoprotein maturation and transport. They are very dynamic organelles, moving in and out of the nucleolus and interacting with each other. They are thought to provide a location where components can be assembled before release to the site of function. Most proteins are in constant motion, and their residence time within a compartment is very low, being at most 1 min [20]. This mobility ensures that proteins find their targets by energy-independent passive diffusion [21]. Given such mobility and the capacity of several small nuclear bodies to self-interact [22], the nuclear architecture is largely driven by a self-organization process [15]. This impressive process can be observed when the compartments disassemble and reassemble during cell division [23]. Hence, the formation of structures in the nucleus is influenced by many molecules, and provides an elegant mechanism not only to concentrate factors when they are needed, but also to segregate factors 


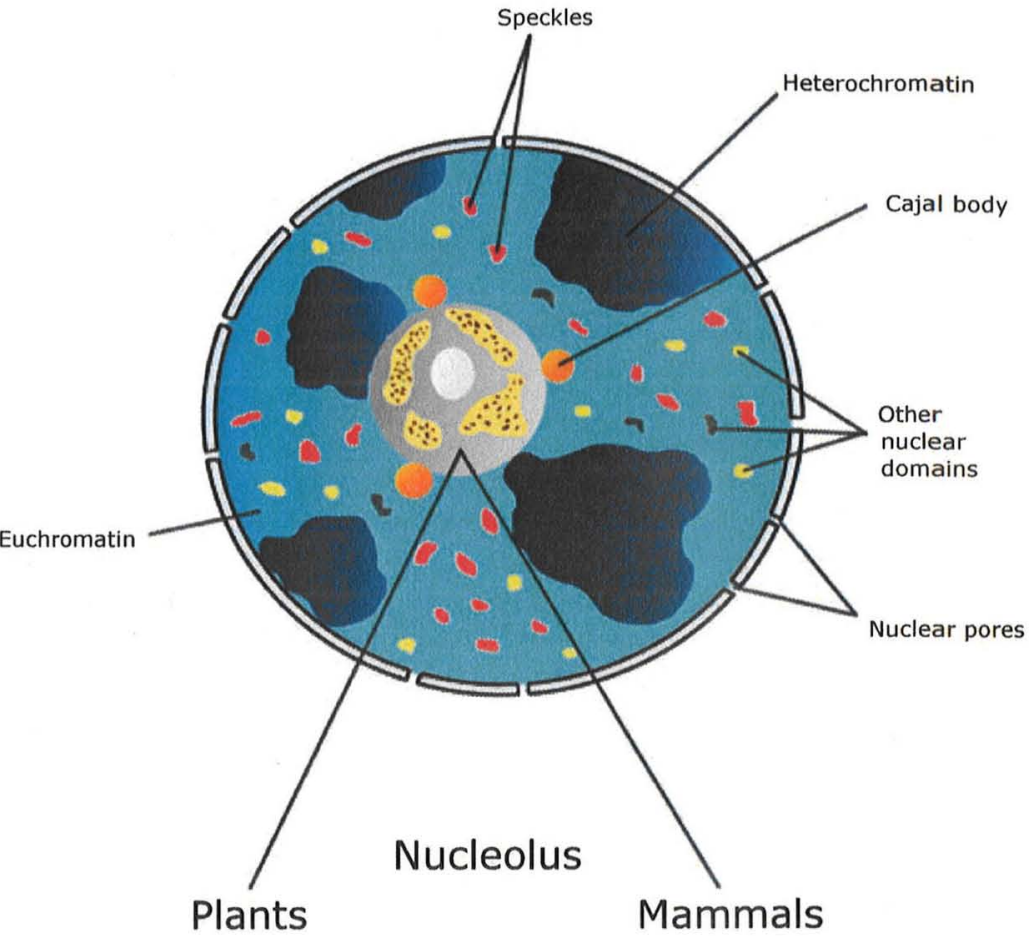

Fig. 1. Schematic presentation of nuclear domains, including heterochromatin and euchromatin entities, CBs, speckles and other domains, as well as a comparison between the nucleolus of mammalian and plant cells. GC, granular component; TS, transcription site.

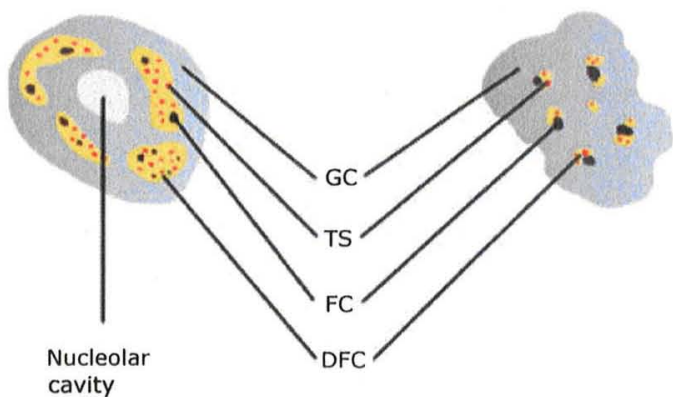

$1 \mu \mathrm{m}$

away from sites where they are debilitating [24]. The movement of molecules is not restricted within the nucleus, and the latest reports suggest that several nuclear proteins have regulatory functions in the whole cell $[25,26]$. The nuclear envelope should not be considered as an insuperable frontier that is simply keeping everything together. It is a double membrane of two lipid bilayers, the outer nuclear membrane being continuous with the endoplasmic reticulum and studded with ribosomes, and the inner membrane hosting a unique complement of integral proteins interacting with chromatin and the nuclear lamina. Both membranes are perforated by large multiprotein complexes, the nuclear pores, which span the entire nuclear envelope and form channels through it, hence opening the border for molecular exchange.
Even though the nuclei of all eukaryotes are very similar in appearance, there are some significant differences between higher plants and mammals, including plant-specific molecular pathways. Unfortunately, very little is known, as yet, about the organization of the plant nucleus and its compartments. Until recently, knowledge about the nucleus in planta was limited to the characterization of the nucleolus, the CBs, and speckles $[1,27]$. Speckles are areas in mammalian cells containing some splicing factors and snRNP proteins. In plants, speckles have been recently shown to contain SR (serine/arginine-rich) proteins. SR proteins constitute a family of splicing factors that contain an RNA-binding motif and an SR region. They form part of the splicosome, being involved in its assembly and participating in intron and exon recognition [28]. 
Nucleoli of mammalian cells, observed by transmission electron microscopy, show three different regions: the fibrillar centres (FCs), which are small, light-staining structures; surrounding the FCs, densely stained material called the dense fibrillar component (DFC); and a region containing many particles, called the granular component, surrounding the DFC. It has been shown that transcription occurs within the DFC [29]. In plant cells, in contrast, the nucleolus is seen to be far more spherical. The DFC is much larger (up to $70 \%$ of the nucleolar volume) and not so dense. Unlike in the mammalian DFC, rDNA transcription units are well dispersed all over the nucleolus, and form structures resembling fir trees, described as 'linear compacted Christmas trees' [30]. These unusual structures have also been reported in HeLa cells, although they harbour a much smaller DFC in these structures [29]. Additionally, there is an eye-catching feature in the centre of the nucleolus, called the nuclear cavity, whose function is still unknown. It has been shown that the nuclear cavity empties itself into the nucleoplasm [31], and that it contains small nuclear RNAs and small nucleolar RNAs [32,33].

CBs are very common particles in nuclei throughout all the different kingdoms. They usually associate with the nucleolus, and seems to be involved in snRNP and small nucleolar ribonucleoprotein maturation. They are thought to provide a location where components can be preassembled before release to the site of function. It has been shown that they are dynamic complexes, moving very fast between the nucleus and the nucleolus [34]. The difference between mammalian and plant cells, in terms of $\mathrm{CBs}$, is simply their presence or absence. Whereas CBs have been observed in every plant nucleus, some mammalian cells lack them. It has been shown that CBs are prominent in cells showing high levels of transcriptional activity but are less abun- dant or absent in some primary cells and tissues [35]. As neither animal nor plant mutants that lack CBs suffer from major losses in vitality, this has led to questions about the function of these particles [1].

All of these findings support the idea of novel, as yet unknown, molecular pathways within the plant nucleus, and strongly support the need for more research in that specific area. However, it should be remembered that obtaining evidence from a model organism rather than the organism of interest can never lead to completely reliable conclusions about the real process, especially when protein interactions are being investigated rather than a single protein. Plants differ greatly in their properties, and this should act as a warning that their molecular interactions may differ as well. Hence, it is always advisable to attempt to perform research using the organism of interest instead of using a related, less difficult to handle model.

\section{Where proteomics join the game}

Investigations of the nucleus were traditionally performed by microscopy, owing to difficulties in biochemical analysis. Today, the ability of MS to identify and to precisely quantify thousands of proteins from complex samples [3] might help to establish protein relationships, especially in organisms with sequenced genomes (http://www.genomesonline.org/), such as A. thaliana [7], Oryza sativa [36,37], Populus trichocarpa [38], and Vitis vinifera [39-41]. Subproteomics of the nucleus and its compartments will further facilitate the annotation of nuclear proteins. There are already several databases available (see Table 1), and these will contribute greatly to improvements in plant cell proteomics. As new proteins are experimentally localized in the nucleus, new software applications such as BACELLO (http://gpcr.biocomp.unibo.it/bacello/) [53]

Table 1. Nuclear protein databases.

\begin{tabular}{|c|c|c|}
\hline Biological source & URL & References \\
\hline \multirow[t]{2}{*}{ Plant transcription factors } & http://plntfdb.bio.uni-potsdam, de/v3.0/ & [42] \\
\hline & http://planttfdb.cbi.pku.edu.cn/ & [43] \\
\hline \multirow[t]{3}{*}{ A. thaliana transcription factors } & http://datf.cbi.pku.edu.cn/ & [44] \\
\hline & http://arabidopsis med.ohio-state.edu/AtTFDB/ & [45] \\
\hline & http://rarge.psc.riken.jp/rartf/ & [46] \\
\hline A. thaliana nucleolar proteins & http://bioinf.scri.sari.ac.uk/cgi-bin/atnopdb/home & [47] \\
\hline Tobacco transcription factors & http://compsysbio.achs.virginia.edu/tobfac/ & [48] \\
\hline Rice transcription factors & http://drtf.cbi.pku.edu.cn/ & [49] \\
\hline Poplar transcription factors & http://dptf.cbi.pku.edu.cn/ & {$[50]$} \\
\hline Grape transcription factors & http://plntfdb.bio.uni-potsdam.de/v3.0/index.php?sp_id=Wla & [43] \\
\hline Soy transcription factors & http://casp.rnet.missouri.edu/soydb/ & {$[51]$} \\
\hline Wheat transcription factors & http://wwwapplinantes.inra fr:8180/WDBFT/ & [52] \\
\hline
\end{tabular}


are being developed and the accuracy of their predictions is increasing. BACELLO can predict the subcellular localization of proteins within five classes (secretory pathway, cytoplasm, nucleus, mitochondrion, and chloroplast) and is based on a decision tree of several support vector machines.

Many studies using subcellular organelles have reported the identification of proteins that were predicted to be localized in other compartments. Hence, intracellular protein trafficking is more complex than believed, and unexpected routes may exist.

Proteomics is a rising field for research on interactions within a cell. Hardly any other technique has the potential to reveal so many details about the cellular state at a single point of time. This is clearly the main advantage, giving scientists the opportunity to observe individual proteins playing their part in the overall scheme.

\section{Current proteomics methods}

Proteomics is now entering its third decade as a field of study. Much of the last two decades was completely dominated by two-dimensional gel electrophoresis (2DE) and usual protein staining techniques as the primary means to conduct comparative experiments. After the many improvements in 2DE technology, its popularization in the $1980 \mathrm{~s}$, and its use in conjunction with MS technology, it definitely became a major tool in a wide range of proteomics research $[54,55]$. One of the main advantages of $2 \mathrm{DE}$ consists of its ability to simultaneously separate and visualize a wide number of proteins [56]. The $2 \mathrm{DE}$ process is based on two autonomous separation methods, the first of which is isoelectric focusing. This process is defined by differently charged proteins being separated by their isoelectric points on an immobilized $\mathrm{pH}$ gradient. The proteins are then transferred to a large SDS/PAGE gel, and separated by their molecular masses. Each 2DE gel generates a protein profile visualized as spots that represent the proteins. The technique has been used for over 30 years, and its reproducibility was clearly improved with the introduction of immobilized $\mathrm{pH}$ gradient gel strips and bioinformatics [56]. This technique is productive in providing relevant data about biological systems. Several authors [25,57-59] have utilized this strategy to investigate plant protein expression in organelles. Nevertheless, problems with sensitivity, throughput and reproducibility of this method place boundaries on comparative proteomics studies, especially in nuclear samples, which have low protein content.

The use of MS is essential for protein identification, and is commonly associated with electrophoretic techniques (Fig. 2). In this area, numerous techniques have been utilized, including MALDI-TOF and ESI $[60,61]$. Furthermore, ion trap and triple-quadruple tandem MS (MS/MS) spectrometers have improved sensitivity and mass accuracy [3]. Finally, some quantitative plant proteomics studies became feasible

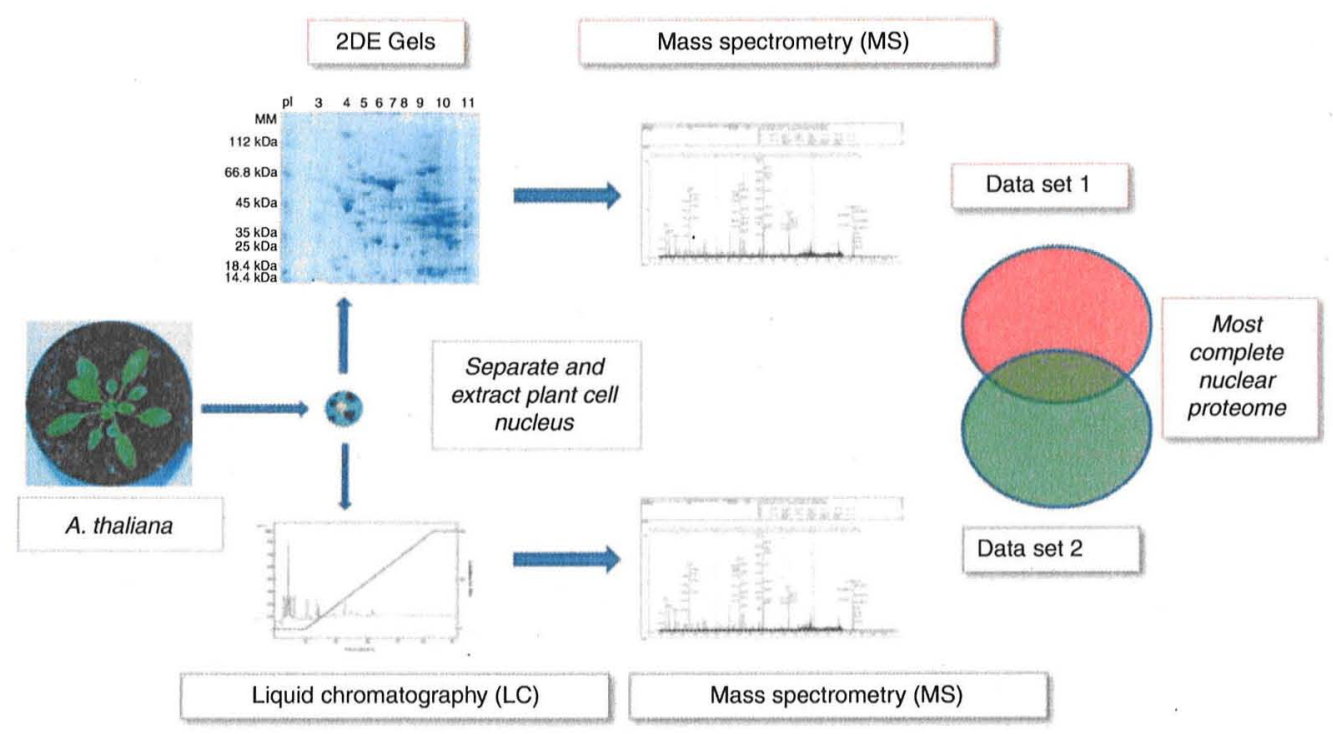

Fig. 2. Synergistic proteomic strategies (gel-free LC/MS and 2-DE/MS) that could be utilised to understand the plant cell nucleus. Circles (green and red) indicate two different data sets of identical sample. The shaded region indicates a possible overlap in these data. 
with the use of an innovative reagent, termed isotopecoded affinity tag, in the liquid chromatography (LC)-MS/MS system [62]. All of these techniques have been applied to plant protein identification in comparative proteomics studies, which have included plant nucleus proteomics [25]. Nevertheless, novel techniques are vital in order to improve data quality at very low sensible levels. These new approaches will be evaluated later in this article.

On the other hand, as pointed out by Jorrin-Novo et al. [8], analytical or biological use of peptidomics, and gel-free, LC-based approaches, including multidimensional protein identification technology [63], could be evaluated for plant nuclear proteomics studies. In summary, multidimensional protein identification technology is a nongel approach for identification of proteins in complex mixtures. The procedure consists of a two-dimensional chromatography separation, followed by electrospray MS. The first dimension is normally a strong cation exchange column, and the second dimension comprises reverse-phase chromatography. The latter is able to remove the salts, and has the added benefit of being compatible with electrospray MS. Such techniques must be applied to nuclear investigations, as it has been observed in the proteomics literature that the different techniques, platforms and workflows are completely complementary (Fig. 2), and that all of them are necessary for complete coverage of the plant nuclear proteome.

\section{An update on A. thaliana nucleus proteomics}

Most large-scale proteomic analyses in Arabidopsis have been carried out with subproteomes (Table 2). Giavalisco et al. [64] designed a large-scale study of the Arabidopsis proteome to achieve complete coverage using 2DE and MALDI-TOF MS. They identified only 663 different proteins from 2943 spots, although a large number of these were found to be expressed as tissue-specific isoforms encoded by different genes.

Until now, an attempt at complete coverage of the A. thaliana nucleus proteome has only been made by Bae et al. [25]. They detected 500-700 spots on 2DE gels, and constructed a $2 \mathrm{DE}$ reference map for nuclear proteins. Analysis by MALDI-TOF MS led to the identification of 184 spots corresponding to 158 different proteins implicated in various cellular functions. This work provided a first view of the complex protein composition in the plant nucleus. To increase the resolution of the 2DE gels, Bae et al. used $\mathrm{pH}$ ranges from 47 and from 6-9. The data indicated that nuclear proteins in basic regions are low in abundance. The
Table 2. Subproteomes of different organelles previously analysed.

\begin{tabular}{ll}
\hline Organelle & Reference \\
\hline Plastids & {$[65,66]$} \\
Mitochondria & {$[67]$} \\
Peroxisomes & {$[68,69]$} \\
Nucleoli & {$[27]$} \\
Cell walls & {$[70-72]$} \\
Vacuoles & {$[73,74]$} \\
Plasma membranes & {$[75]$} \\
Cytosolic ribosomes & {$[76]$} \\
\hline
\end{tabular}

identification of 54 proteins upregulated or downregulated in response to cold stress indicates a major regulatory function of the nucleus. The control of gene expression occurs largely at the transcriptional or posttranscriptional levels. It seems that proteins implicated in signalling and gene regulation dominate each other. This is in contrast to what has been found in the analysis of other organelles [57,59], supporting the importance of the nucleus in cell regulation. After all, Bae et al. [25] have shown that a complex mechanism underlies the response to stress and that several cellular functions are, at least partially, controlled by proteins emerging from the nucleus.

Whereas there have been plenty of data published concerning the human nucleolus [77-79], information about the nucleolus in plants is still very limited. In 2005, Pendle et al. [27] published the first proteomic analysis of $A$. thaliana nucleoli. The authors identified 217 proteins, many of which many could be compared to those in the proteome of human nucleoli. Proteins with the same function in humans, plant-specific proteins, proteins of unknown function and some that are nucleolar in plants, but non-nucleolar in humans, were found. Interestingly, Pendle et al. identified six components of the postsplicing exon-junction complex involved in mRNA export and nonsense-mediated decay/mRNA surveillance, raising the possibility that plant nucleoli may be involved in mRNA export and surveillance. Of the proteins described by Pendle et al. [27], $69 \%$ have a direct counterpart in animals, whereas up to $30 \%$ of the nucleolar proteins are encoded by new, as yet uncharacterized, genes [78,80]. This further supports the importance of comparative proteomics approaches between Arabidopsis and human nucleoli.

Analysis of the nuclear matrix by $2 \mathrm{DE}$ and MS by Calikowski et al. [17] resolved approximately 300 protein spots, including Arabidopsis homologues of nucleolar proteins, ribosomal components, and a putative histone deacetylase. There were homologues of the human nuclear matrix and nucleolar proteins, as well 
as novel proteins with unknown functions. The identification of 36 proteins by MS demonstrated that several classes of functional protein in the nuclear matrix are shared between vertebrates and higher plants, and that there is great enrichment of proteins associated with the nucleolus [23].

Recently, Jones et al. [81] enriched nuclei from Arabidopsis cell cultures and seedlings. Within those, they identified 416 phosphopeptides from 345 proteins, including novel phosphorylation sites and kinase motifs on transcription factors, chromatin-remodelling proteins, RNA-silencing components, and the splicosome. Phosphorylation is a crucial process for intramolecular and intermolecular interactions, as it directly alters protein activity. Identification of the phosphorylation sites is an important step towards the understanding of protein interaction within the nucleus and its function as a cellular regulator.

\section{An update on $O$. sativa nucleus proteomics}

Whereas A. thatiana is clearly the most thoroughly explored plant for nuclear proteomics research, there are several groups working with other species. As stated earlier, comparative analyses are of major importance for a complete understanding of nuclear proteomics. It is therefore mandatory to include other species in nuclear research. $O$. sativa is, without doubt, one of the most important crops to be investigated, considering its worldwide nutritional importance. In any case, Oryza suits proteomics research very well, being a very easily grown plant. Developments in rice nuclear proteomics were reviewed by Khan et al. [82], and will not be discussed in detail here. Briefly, they discovered 549 proteins and identified 190 of them by database searching. Most of these proteins were found to be involved mainly in signalling and gene regulation, supporting the role of the nucleus in cellular regulation. This is in agreement with the findings of Bae et al. [25] in Arabidopsis nuclei. In 2007, Tan et al. [83] published data on proteomic and phophoproteomic analysis and chromatinassociated proteins in Oryza. They found 509 proteins by MS, corresponding to 269 unique proteins, including nucleosome assembly proteins, high-mobility group proteins, histone modification proteins, transcription factors, and a large number of proteins of unknown function. In addition, they found 128 chromatin-associated proteins, using a shotgun approach. Interestingly, they observed a large number of histone variants in rice, e.g. 11 variants of histone $\mathrm{H} 2 \mathrm{~A}$, whereas only six variants of histone $\mathrm{H} 2 \mathrm{~A}$ are known in mammals [84]. Specific histone variants in the nucleosome are known to generate distinct chromosomal domains for the regulation of gene expression [84,85]. More recently, however, Aki et al. [86] reported 657 proteins in rice nuclei, among them novel nuclear factors involved in evolutionarily conserved mechanisms for sugar responses in the plant. They proposed two WD40-like proteins and one armadillo/pumilio-like protein as candidates for such nuclear factors. This is particularly interesting, as sugar is one of the key regulators of development in both plants and animals. Another recent publication by Choudhary et al. [58] described the response of the rice nucleus to dehydration. They found 150 spots on $2 \mathrm{DE}$ gels that displayed changes in their intensities by up to 2.5-fold when exposed to stress. Among them, they identified 109 proteins with various functions, including cellular regulation, protein degradation, cellular defence, chromatin remodelling, and transcriptional regulation. All of these findings further support the role of the nucleus as the main cellular regulator.

\section{An update on Cicer arietinum and Medicago truncatula nucleus proteomics}

Besides those groups working on the quite common plant species $A$. thaliana and $O$. sativa, there are other groups using more unusual plants as their model organisms. In 2006, Panday et al. [87] published the first report of the nuclear proteome of the as yet unsequenced genome of the chickpea $C$. arietinum. They resolved approximately 600 proteins on $2 \mathrm{DE}$ gels, and identified 150 of them. The found a variety of different protein classes; the largest number of proteins was involved in signalling and gene regulation (36\%), followed by DNA replication and transcription (17\%). Overall, they grouped the proteins into 10 different classes with completely different functions. Additionally, they attempted to compare the proteomes of Arabidopsis, rice, and chickpea. They found only eight identical proteins in all three organisms; these were some of the 32 common proteins in Arabidopsis and chickpea. Chickpea and rice shared 11 proteins, whereas rice and Arabidopsis had only six proteins in common. They stated that $71 \%$ of the chickpea nuclear proteins are novel, demanding further research for a better understanding of the nuclear proteome of plants. In 2008, the same group published the first proteomics approach to identify dehydration-responsive nuclear proteins from chickpea [88]. Dehydration is one of the most common environmental stresses, being 
caused not only by the absence of water in the soil or excessive heat, but also intracellular ice during freezing. They found 205 spots on $2 \mathrm{DE}$ gels that changed their intensities by more than 2.5 -fold under dehydration stress; 80 of them were upregulated, 46 were downregulated, and 79 showed time-dependent mixed expression. Of these proteins, 147 were subjected to MS/MS analysis, resulting in the identification of 105 proteins. Additionally, they described different isoelectric species of several proteins, probably resulting from post-translational modifications, which are known to affect protein activity. The dehydration stress response within the nucleus seems to be very complex. Several proteins were identified that play a role in early responsive signalling, including, among others, two upregulated histones, histone $\mathrm{H} 3 \quad(\mathrm{CaN}-574)$ and histone $\mathrm{H} 2 \mathrm{~B}(\mathrm{CaN}-575)$, which is interesting, as Tan et al. [83] reported 11 different histone variants in rice nuclei. In summary, the proteins were grouped into 10 classes; the most abundant proteins belonged to the class of gene transcription and replication, closely followed by molecular chaperones. The data collected by Pandey et al. [88] provide a first insight into the molecular changes within the nucleus of the chickpea, and will be of great value for comparison with other plant species.

In 2008, an interesting paper was published by Repetto et al. [89], concerning the nuclear proteome of another legume, M. truncatula, at the switch towards seed filling. Germination and subsequent plant growth are totally dependent on the composition of the seed. Hence, these early steps during seed filling are of upmost importance for the plant. They found that nuclei store a pool of ribosomal proteins in preparation for intense protein synthesis at this stage. Several proteins involved in ribosomal subunit synthesis, transcriptional regulation, chromatin organization and RNA processing, transport and silencing have been identified. Overall, they identified 143 different proteins, and compared them to those in seedling and leaf nuclear proteomes [25,87]. The majority were, as expected, involved in gene regulation. However, they found that proteins involved in DNA metabolism, RNA processing and ribosome biogenesis are more abundant in seed nuclei than in nuclei of leaves or seedlings. They described several novel nuclear proteins involved in the biogenesis of ribosomal subunits (pescadillo-like) or in nucleocytoplasmic trafficking (dynamin-like GTPase). Their data also indicate that, at the switch towards seed filling, the nucleus already contains ribosomal proteins that will be used to form the cytosolic ribosomes for reserve synthesis, and that the genome architecture may be extensively modified during seed development.

\section{Differential proteomics techniques - novel strategies to elucidate the plant cell nucleus}

Numerous important scientific questions concerning the cell nucleus have still not been answered, in spite of the use of common proteomics techniques such as $2 \mathrm{DE}$ and MS identification. In summary, these problems arise from the low sample quantity and low protein concentration. The sensitive detection of peptides and proteins is an enormous challenge, not only in plant cell mucleus proteomics, but also in other fields of biological science. For complete exploitation of this system sensitivity, different purification methods have been proposed, including ultrafiltration, dialysis, and protein precipitation. Moreover, the utilization of magnetic particles as a purification protein tool could be a useful strategy for protein nucleus analyses, as they show clear biochemical properties and also low concentrations [90]. In this interesting article [90], the authors proposed an elegant strategy to improve protein concentration by the addition of magnetic reversed-phase particles to a protein extract. Hydrophobic proteins were attached to particles and recovered with a magnet. The solution was then discarded, the magnetic beds were washed, and the proteins were eluted and subjected to capillary reverse-phase chromatography combined with MALDI-TOF MS for protein identification [90]. Because of the magnetic core, this kind of sample preparation could be automated by using robots, reducing handling mistakes.

However, differential sample preparation could be only part of the solution. More sensitive proteomics techniques are essential to study low quantities of proteins and peptides from plant cell nuclei. In this field, top-down proteomics has emerged as a powerful technique for protein analyses, and is a growing research area in the proteomics community. The most common strategy for top-down proteomic analyses includes the front-end separation of undamaged proteins, their detection and further fragmentation in a mass spectrometer, and a final identification by using the sequence information obtained from MS and MS/MS spectral data [91,92]. Recently, the top-down approach was used to evaluate multiple modifications of histones, including methylation and acetylation [93], suggesting that this approach could also be a valuable tool with which to elucidate several points of plant nucleus control. Quantitative top-down proteomics frequently utilizes stable isotope labelling in order to create an inner standard from which consistent quantitative data may be obtained. For this, stable isotope labelling by amino acids (SILAC) was successfully 
introduced in cell culture [94], creating a new method for quantifying proteins and peptides, whereby amino acids labeled with stable isotopes are supplemented to cell culture broth, with the aim of producing coeluting labeled and unlabelled analytes. Labelled arginine and lysines are commonly used in bottom-up experiments, in conjunction with trypsinization, creating an excellent environment in which to, after mathematical and computational analyses, quantify certain groups of plant nuclear proteins. Recently, a novel extension of the label-chase concept was developed, by using a multitagging proteomics strategy, combining SILAC and a secondary labelling step with ITRAQ reagents, in order to estimate protein turnover rates in fungi [95]. An understanding of the rate of protein production/degradation is indispensable for an understanding of plant nuclear dynamics, and to fill the information gap between transcriptome and proteome. Another approach, in addition to SILAC, consists of the use of MS, electron capture dissociation and electron transfer dissociation to evaluate some post-transcriptional modifications, as obtained for the phosphoproteome of histone H4 [96]. This kind of approach could be extremely valuable for plant nuclear proteome analyses, as phosphorylation seems to be essential for different nuclear processes in plants.

Finally, and no less importantly, bioinformatics seems to be the other challenge for plant proteomics studies. In last few years, several institutions all over the world have established core proteomics facilities to offer MS services. With the increasing requirements for high-throughput analyses of complex samples and the enhanced interest in quantitative proteomics, effective data analysis may be a real challenge. Several efforts have been made in this direction, including the Central Proteomics Facilities Pipeline [97]. This server offers identification, validation and quantitative analyses of proteins and peptides from LC-MS/MS datasets by web interface, facilitating all analyses for the researcher. This kind of approach could clearly facilitate the identification of specific nuclear proteins. Moreover, once that the understanding of the plant nucleus is directly related to the knowledge of several biological processes and those processes involve different proteins that act synergistically, an in silico active learning approach for protein-protein interaction prediction is also indicated to learn more about the plant nucleus. In this view, random forest has been previously shown to be effective for the prediction of protein-protein interactions in humans [98], indicating that this active-learning algorithm enables more accurate protein classification. In summary, the future of plant nucleus proteomics is probably related to novel
MS technologies associated with novel in silico approaches, which could improve the rate of acquisition, quantity and quality of proteomics data provided.

\section{Conclusions}

Without doubt, we are on the brink of a postgenomic era in plant research. The completion of $A$. thaliana genome sequencing emphasized the importance of high-throughput analysis approaches. We can now focus on understanding the complex relationships between molecules and their involvement in cell regulation. The subproteome of the nucleus might play only a small part in that, but it has been made clear that this awe-inspiring organelle could be more involved in the overall cellular estate than imagined. The data presented on the latest attempts to cover the nuclear proteome of several plant species are of great value. Besides the expected, there have been several new findings, including proteins of still unknown function, proteins that were not expected to be localized in the nucleus, and completely novel proteins. However, independently of the plant species, the majority of discovered proteins were found to be involved in gene regulation and signalling. Thus, in summary, the data have further supported the role of the plant nucleus as the major cellular regulator, in the mould of a cell maestro. Not only will $A$. thaliana researchers be able to benefit from a better understanding of the nucleus, but the latest data have also shown many counterparts of mammalian proteins, as well as proteins of unknown function. Direct comparison with the most sought-after proteins, e.g. those that have been shown to enhance cancer in human cells, has to be handled with care, although some similarities may be present and support further studies. Hence, the possibility of intra-kingdom or cross-kingdom comparison of not only some random proteins but real cellular regulation schemes with the use of advanced proteomics techniques is of great value to anyone working in the molecular field. We have the tools in our hands. All we need to do now is to combine the different fields of research to reach a new level of understanding.

\section{References}

1 Shaw PJ \& Brown JW (2004) Plant nuclear bodies. Curr Opin Plant Biol 7, 614-620.

2 Misteli T (2009) Self-organization in the genome. Proc Natl Acad Sci USA 106, 6885-6886.

3 Aebersold R \& Mann M (2003) Mass spectrometrybased proteomics. Nature 422, 198-207. 
4 Trinkle-Mulcahy L \& Lamond AI (2007) Toward a high-resolution view of nuclear dynamics. Science 318, 1402-1407.

5 Jung E, Heller M, Sanchez JC \& Hochstrasser DF (2000) Proteomics meets cell biology: the establishment of subcellular proteomes. Electrophoresis 21, 3369-3377.

6 Baginsky S \& Gruissem W (2006) Arabidopsis thaliana proteomics: from proteome to genome. $J$ Exp Bot 57, 1485-1491.

7 The Arabidopsis Genome Initiative (2000) Analysis of the genome sequence of the flowering plant Arabidopsis thaliana. Nature 408, 796-815.

8 Jorrin-Novo JV, Maldonado AM, Echevarria-Zomeno $\mathrm{S}$, Valledor L, Castillejo MA, Curto M, Valero J, Sghaier B, Donoso G \& Redondo I (2009) Plant proteomics update (2007-2008): second-generation proteomic techniques, an appropriate experimental design, and data analysis to fulfill MIAPE standards, increase plant proteome coverage and expand biological knowledge.

$J$ Proteomics 72, 285-314.

9 Jorrin-Novo JV (2009) Plant proteomics. J Proteomics 72, 283-284.

10 Park OK (2004) Proteomic studies in plants. $J$ Biochem Mol Biol 37, 133-138.

11 Kersten B, Burkle L, Kuhn EJ, Giavalisco P, Konthur Z, Lueking A, Walter G, Eickhoff $\mathrm{H} \&$ Schneider U (2002) Large-scale plant proteomics. Plant Mol Biol 48, 133-141.

12 Zivy M \& de Vienne D (2000) Proteomics: a link between genomics, genetics and physiology. Plant Mol Biol 44, 575-580.

13 van Wijk KJ (2001) Challenges and prospects of plant proteomics. Plant Physiol 126, 501-508.

14 Dundr M \& Misteli T (2001) Functional architecture in the cell nucleus. Biochem $J$ 356, 297-310.

15 Misteli T (2001) The concept of self-organization in cellular architecture. $J$ Cell Biol 155, 181-185.

16 Nickerson J (2001) Experimental observations of a nuclear matrix. $J$ Cell Sci 114, 463-474.

17 Calikowski TT, Meulia T \& Meier I (2003) A proteomic study of the arabidopsis nuclear matrix. $J$ Cell Biochem 90, 361-378.

18 Cremer T, Kreth G, Koester H, Fink RH, Heintzmann R, Cremer M, Solovei I, Zink D \& Cremer C (2000) Chromosome territories, interchromatin domain compartment, and nuclear matrix: an integrated view of the functional nuclear architecture. Crit Rev Eukaryot Gene Expr 10, 179-212.

19 Holmes-Davis R (1998) Nuclear matrix attachment regions and plant gene expression. Trends Plant Sci 3 91-97.

20 Gonzalez-Melendi P, Beven A, Boudonck K, Abranches R, Wells B, Dolan L \& Shaw P (2000) The nucleus: a highly organized but dynamic structure. J Microsc 198, 199-207.
21 Pederson T (2000) Diffusional protein transport within the nucleus: a message in the medium. Nat Cell Biol 2, E73-74.

22 Hebert MD \& Matera AG (2000) Self-association of coilin reveals a common theme in nuclear body localization. Mol Biol Cell 11, 4159-4171.

23 Dundr M, Misteli T \& Olson MO (2000) The dynamics of postmitotic reassembly of the nucleolus. $J$ Cell Biol 150, 433-446.

24 Misteli T (2000) Cell biology of transcription and premRNA splicing: nuclear architecture meets nuclear function. $J$ Cell Sci 113(Pt 11), 1841-1849.

25 Bae MS, Cho EJ, Choi EY \& Park OK (2003) Analysis of the Arabidopsis nuclear proteome and its response to cold stress. Plant $J$ 36, 652-663.

26 Wilkie GS \& Schirmer EC (2006) Guilt by association: the nuclear envelope proteome and disease. $\mathrm{Mol} \mathrm{Cell}$ Proteomics 5, 1865-1875.

27 Pendle AF, Clark GP, Boon R, Lewandowska D, Lam YW, Andersen J, Mann M, Lamond AI, Brown JW \& Shaw PJ (2005) Proteomic analysis of the Arabidopsis nucleolus suggests novel nucleolar functions. $\mathrm{Mol}$ Biol Cell 16, 260-269.

28 Graveley BR (2000) Sorting out the complexity of SR protein functions. RNA 6, 1197-1211.

29 Koberna K, Malinsky J, Pliss A, Masata M, Vecerova J, Fialova M, Bednar J \& Raska I (2002) Ribosomal genes in focus: new transcripts label the dense fibrillar components and form clusters indicative of "Christmas trees' in situ. $J$ Cell Biol 157, 743-748.

30 Gonzalez-Melendi P, Wells B, Beven AF \& Shaw PJ (2001) Single ribosomal transcription units are linear, compacted Christmas trees in plant nucleoli. Plant $J \mathbf{2 7}$, 223-233.

31 Gunning BES (2004) Plant Cell Biology on CD - information for students and a resource for teachers, Part 1 . www.plantcellbiologyonCD.com. Accessed July 05, 2010.

32 Beven AF, Simpson GG, Brown JW \& Shaw PJ (1995) The organization of spliceosomal components in the nuclei of higher plants. $J$ Cell Sci $108(\mathrm{Pt} 2)$, 509-518.

33 Beven AF, Lee R, Razaz M, Leader DJ, Brown JW \& Shaw PJ (1996) The organization of ribosomal RNA processing correlates with the distribution of nucleolar snRNAs. J Cell Sci 109(Pt 6), 1241-1251.

34 Platani M, Goldberg I, Swedlow JR \& Lamond AI (2000) In vivo analysis of Cajal body movement, separation, and joining in live human cells. $J$ Cell Biol 151, $1561-1574$

35 Ogg SC \& Lamond AI (2002) Cajal bodies and coilin moving towards function. $J$ Cell Biol 159, 17-21.

$36 \mathrm{Yu} \mathbf{J}$, Hu S, Wang J, Wong GK, Li S, Liu B, Deng Y, Dai L, Zhou Y, Zhang X et al. (2002) A draft sequence of the rice genome (Oryza sativa L. ssp. indica). Science 296, 79-92. 
37 Goff SA, Ricke D, Lan TH, Presting G, Wang R, Dunn M, Glazebrook J, Sessions A, Oeller P, Varma H et al. (2002) A draft sequence of the rice genome (Oryza sativa L. ssp. japonica). Science 296, 92-100.

38 Tuskan GA, Difazio S, Jansson S, Bohlmann J, Grigoriev I, Hellsten U, Putnam N, Ralph S, Rombauts S, Salamov A et al. (2006) The genome of black cottonwood, Populus trichocarpa (Torr. \& Gray). Science 313, 1596-1604.

39 Jaillon O, Aury JM, Noel B, Policriti A, Clepet C, Casagrande A, Choisne N, Aubourg S, Vitulo N, Jubin $C$ et al. (2007) The grapevine genome sequence suggests ancestral hexaploidization in major angiosperm phyla. Nature 449, 463-467.

40 Velasco R, Zharkikh A, Troggio M, Cartwright DA, Cestaro A, Pruss D, Pindo M, Fitzgerald LM, Vezzulli $\mathrm{S}$, Reid $\mathrm{J}$ et al. (2007) A high quality draft consensus sequence of the genome of a heterozygous grapevine variety. PLOS ONE 2, el 326.

41 Peck SC (2005) Update on proteomics in Arabidopsis. Where do we go from here?. Plant Physiol 138, 591599.

42 Perez-Rodriguez P, Riano-Pachon DM, Correa LG, Rensing SA, Kersten B \& Mueller-Roeber B (2010) PInTFDB: updated content and new features of the plant transcription factor database. Nucleic Acids Res 38, D822-827.

43 Guo AY, Chen X, Gao G, Zhang H, Zhu QH, Liu XC, Zhong YF, Gu X, He K \& Luo J (2008) PlantTFDB: a comprehensive plant transcription factor database. Nucleic Acids Res 36, D966-969.

44 Guo A, He K, Liu D, Bai S, Gu X, Wei L \& Luo J (2005) DATF: a database of Arabidopsis transcription factors. Bioinformatics 21, 2568-2569.

45 Palaniswamy SK, James S, Sun H, Lamb RS, Davuluri RV \& Grotewold E (2006) AGRIS and AtRegNet. a platform to link cis-regulatory elements and transcription factors into regulatory networks. Plant Physiol 140, 818-829.

46 Iida K, Seki M, Sakurai T, Satou M, Akiyama K, Toyoda T, Konagaya A \& Shinozaki K (2005) RARTF: database and tools for complete sets of Arabidopsis transcription factors. DNA Res 12, 247-256.

47 Brown JW, Shaw PJ, Shaw P \& Marshall DF (2005) Arabidopsis nucleolar protein database (AtNoPDB). Nucleic Acids Res 33, D633-636.

48 Rushton PJ, Bokowiec MT, Laudeman TW, Brannock JF, Chen X \& Timko MP (2008) TOBFAC: the database of tobacco transcription factors. BMC Bioinformatics 9, 1-7.

49 Gao G, Zhong Y, Guo A, Zhu Q, Tang W, Zheng W, Gu X, Wei L \& Luo J (2006) DRTF: a database of rice transcription factors. Bioinformatics 22, 1286-1287.

50 Zhu QH, Guo AY, Gao G, Zhong YF, Xu M, Huang M \& Luo J (2007) DPTF: a database of poplar transcription factors. Bioinformatics 23, 1307-1308.
51 Wang Z, Libault M, Joshi T, Valliyodan B, Nguyen HT, Xu D, Stacey G \& Cheng J (2010) SoyDB: a knowledge database of soybean transcription factors. BMC Plant Biol 10, 1-12.

52 Romeuf $\mathrm{l}$, Tessier D, Dardevet M, Branlard G, Charmet G \& Ravel C (2010) wDBTF: an integrated database resource for studying wheat transcription factor families. BMC Genomics 11, 1-15.

53 Pierleoni A, Martelli PL, Fariselli P \& Casadio R (2006) BaCelLo: a balanced subcellular localization predictor. Bioinformatics 22, e408-416.

54 Lee J, Garrett WM \& Cooper B (2007) Shotgun proteomic analysis of Arabidopsis thaliana leaves. $J$ Sep Sci 30, 2225-2230.

55 Westermeier R \& Marouga R (2005) Protein detection methods in proteomics research. Biosci Rep 25, 19-32.

56 Gorg A, Obermaier C, Boguth G, Harder A, Scheibe B, Wildgruber R \& Weiss W (2000) The current state of two-dimensional electrophoresis with immobilized $\mathrm{pH}$ gradients. Electrophoresis 21, 1037-1053.

57 Millar AH, Sweetlove LJ, Giege P \& Leaver CJ (2001) Analysis of the Arabidopsis mitochondrial proteome. Plant Physiol 127, 1711-1727.

58 Choudhary MK, Basu D, Datta A, Chakraborty N \& Chakraborty S (2009) Dehydration-responsive nuclear proteome of rice (Oryza sativa L.) illustrates protein network, novel regulators of cellular adaptation, and evolutionary perspective. Mol Cell Proteomics 8, 1579-1598.

59 Peltier JB, Friso G, Kalume DE, Roepstorff P, Nilsson F, Adamska I \& van Wijk KJ (2000) Proteomics of the chloroplast: systematic identification and targeting analysis of lumenal and peripheral thylakoid proteins. Plant Cell 12, 319-341.

60 Fenn JB, Mann M, Meng CK, Wong SF \& Whitehouse CM (1989) Electrospray ionization for mass spectrometry of large biomolecules. Science 246, 64-71.

61 Karas M \& Hillenkamp F (1988) Laser desorption ionization of proteins with molecular masses exceeding 10,000 daltons. Anal Chem 60, 2299-2301

62 Han DK, Eng J, Zhou H \& Aebersold R (2001) Quantitative profiling of differentiation-induced microsomal proteins using isotope-coded affinity tags and mass spectrometry. Nat Biotechnol 19, 946-951.

63 Jain R, Katavic V, Agrawal GK, Guzov VM \& Thelen JJ (2008) Purification and proteomic characterization of plastids from Brassica napus developing embryos. Proteomics 8, 3397-3405.

64 Giavalisco P, Nordhoff E, Kreitler T, Kloppel KD, Lehrach H, Klose J \& Gobom J (2005) Proteome analysis of Arabidopsis thaliana by two-dimensional gel electrophoresis and matrix-assisted laser desorption/ ionisation-time of flight mass spectrometry. Proteomics 5, 1902-1913.

65 van Wijk KJ (2004) Plastid proteomics. Plant Physiol Biochem 42, 963-977. 
66 Baginsky S \& Gruissem W (2004) Chloroplast proteomics: potentials and challenges. $J$ Exp Bot 55, 12131220.

67 Millar AH, Heazlewood JL, Kristensen BK, Braun HP \& Moller IM (2005) The plant mitochondrial proteome. Trends Plant Sci 10, 36-43.

68 Fukao Y, Hayashi M \& Nishimura M (2002) Proteomic analysis of leaf peroxisomal proteins in greening cotyledons of Arabidopsis thaliana. Plant Cell Physiol 43, 689-696.

69 Palma JM, Corpas FJ \& del Rio LA (2009) Proteome of plant peroxisomes: new perspectives on the role of these organelles in cell biology. Proteomics 9, 23012312.

70 Chivasa S, Ndimba BK, Simon WJ, Robertson D, Yu XL, Knox JP, Bolwell P \& Slabas AR (2002) Proteomic analysis of the Arabidopsis thaliana cell wall. Electrophoresis 23, 1754-1765.

71 Borderies G, Jamet E, Lafitte C, Rossignol M, Jauneau A, Boudart G, Monsarrat B, Esquerre-Tugaye MT, Boudet A \& Pont-Lezica R (2003) Proteomics of loosely bound cell wall proteins of Arabidopsis thaliana cell suspension cultures: a critical analysis. Electrophoresis 24, 3421-3432.

72 Boudart G, Jamet E, Rossignol M, Lafitte C, Borderies G, Jauneau A, Esquerre-Tugaye MT \& Pont-Lezica R (2005) Cell wall proteins in apoplastic fluids of Arabidopsis thaliana rosettes: identification by mass spectrometry and bioinformatics. Proteomics 5 , 212-221.

73 Carter C, Pan S, Zouhar J, Avila EL, Girke T \& Raikhel NV (2004) The vegetative vacuole proteome of Arabidopsis thaliana reveals predicted and unexpected proteins. Plant Cell 16, 3285-3303.

74 Shimaoka T, Ohnishi M, Sazuka T, Mitsuhashi N, Hara-Nishimura I, Shimazaki K, Maeshima M, Yokota A, Tomizawa K \& Mimura T (2004) Isolation of intact vacuoles and proteomic analysis of tonoplast from suspension-cultured cells of Arabidopsis thaliana. Plant Cell Physiol 45, 672-683.

75 Ephritikhine G, Ferro M \& Rolland N (2004) Plant membrane proteomics. Plant Physiol Biochem 42, 943962.

76 Chang IF, Szick-Miranda K, Pan S \& Bailey-Serres J (2005) Proteomic characterization of evolutionarily conserved and variable proteins of Arabidopsis cytosolic ribosomes. Plant Physiol 137, 848-862.

77 Lam YW, Trinkle-Mulcahy L \& Lamond Al (2005) The nucleolus. $J$ Cell Sci 118, 1335-1337.

78 Andersen JS, Lam YW, Leung AK, Ong SE, Lyon CE, Lamond AI \& Mann M (2005) Nucleolar proteome dynamics. Nature 433, 77-83.

79 Coute Y, Burgess JA, Diaz JJ, Chichester C, Lisacek F, Greco A \& Sanchez JC (2006) Deciphering the human nucleolar proteome. Mass Spectrom Rev 25, 215-234.
80 Andersen JS, Lyon CE, Fox AH, Leung AK, Lam YW, Steen H, Mann M \& Lamond AI (2002) Directed proteomic analysis of the human nucleolus. Curr Biol $12,1-11$.

81 Jones AM, MacLean D, Studholme DJ, Serna-Sanz A, Andreasson E, Rathjen JP \& Peck SC (2009) Phosphoproteomic analysis of nuclei-enriched fractions from Arabidopsis thaliana. $J$ Proteomics 72, 439-451.

82 Khan MM \& Komatsu S (2004) Rice proteomics: recent developments and analysis of nuclear proteins. Phytochemistry 65, 1671-1681.

83 Tan F, Li G, Chitteti BR \& Peng Z (2007) Proteome and phosphoproteome analysis of chromatin associated proteins in rice (Oryza sativa). Proteomics 7, 4511-4527.

84 Bernstein E \& Hake SB (2006) The nucleosome: a little variation goes a long way. Biochem Cell Biol 84, 505517.

85 Hall DA, Zhu H, Zhu X, Royce T, Gerstein M \& Snyder M (2004) Regulation of gene expression by a metabolic enzyme. Science 306, 482-484.

86 Aki T \& Yanagisawa S (2009) Application of rice nuclear proteome analysis to the identification of evolutionarily conserved and glucose-responsive nuclear proteins. J Proteome Res 8, 3912-3924.

87 Pandey A, Choudhary MK, Bhushan D, Chattopadhyay A, Chakraborty S, Datta A \& Chakraborty N (2006) The nuclear proteome of chickpea (Cicer arietinum L.) reveals predicted and unexpected proteins. $J$ Proteome Res 5, 3301-3311.

88 Pandey A, Chakraborty S, Datta A \& Chakraborty N (2008) Proteomics approach to identify dehydration responsive nuclear proteins from chickpea (Cicer arietinum L.). Mol Cell Proteomics 7, 88-107.

89 Repetto O, Rogniaux H, Firnhaber C, Zuber H, Kuster H, Larre C, Thompson R \& Gallardo K (2008) Exploring the nuclear proteome of Medicago truncatula at the switch towards seed filling. Plant $J$ 56, 398-410.

90 Peter JF \& Otto AM (2010) Magnetic particles as powerful purification tool for high sensitive mass spectrometric screening procedures. Proteomics 10, 628-633.

91 Garcia BA (2010) What does the future hold for Top Down mass spectrometry? J Am Soc Mass Spectrom 21, 193-202.

92 Collier TS, Sarkar P, Rao B \& Muddiman DC (2010) Quantitative top-down proteomics of SILAC labelled human embryonic stem cells. J Am Soc Mass Spec 21, 879-889.

93 Pesavento JJ, Bullock CR, LeDuc RD, Mizzen CA \& Kelleher NL (2008) Combinatorial modification of human histone $\mathrm{H} 4$ quantitated by two-dimensional liquid chromatography coupled with top down mass spectrometry. $J$ Biol Chem 283, 14927-14937.

94 Ong SE, Blagoev B, Kratchmarova I, Kristensen DB, Steen H, Pandey A \& Mann M (2002) Stable isotope labeling by amino acids in cell culture, SILAC, as a 
simple and accurate approach to expression proteomics. Mol Cell Proteomics 1, 376-386.

95 Jayapal KP, Sui S, Philp RJ, Kok YJ, Yap MG, Griffin TJ \& Hu WS (2010) Multitagging Proteomic Strategy to Estimate Protein Turnover Rates in Dynamic Systems. J Proteome Res 9, 2087-2097.

96 Phanstiel D, Brumbaugh J, Berggren WT, Conard K, Feng X, Levenstein ME, McAlister GC, Thomson JA \& Coon JJ (2008) Mass spectrometry identifies and quantifies 74 unique histone $\mathrm{H} 4$ isoforms in differentiating human embryonic stem cells. Proc Natl Acad Sci USA 105, 4093-4098.

97 Trudgian DC, Thomas B, McGowan SJ, Kessler BM, Salek M \& Acuto O (2010) CPFP: a central proteomics facilities pipeline. Bioinformatics $\mathbf{2 6}$, 1131-1132.

98 Mohamed TP, Carbonell JG \& Ganapathiraju MK (2010) Active learning for human protein-protein interaction prediction. BMC Bioinformatics 11(Suppl 1), 1-9. 